\title{
INDUCED AND PRODUCED REPRESENTATIONS OF LIE ALGEBRAS
}

\author{
BY \\ ROBERT J. BLATTNER( $\left.{ }^{1}\right)$
}

1. Introduction. D. G. Higman, in [6], introduced and studied the notions of induced and produced modules of rings. These concepts are generalizations of the classical construction of induced representations of finite groups. In the present paper, we study these notions in the context of modules over Lie algebras (or equivalently unitary modules over the universal enveloping algebras of these Lie algebras).

Induced representations of Lie algebras have come up previously in the work of Harish-Chandra ([4, Theorem 4] and [5, Theorem 1]) and Dixmier ([2] and [2a]), where they were used to study representations of semisimple (resp. nilpotent and solvable) Lie algebras. A recent paper of N. R. Wallach [12] uses produced representations to prove the Borel-Weil theorem. Our interest in the subject is two-fold: to attempt to prove analogues of theorems on induced group representations, and to clarify certain concepts arising from the work of Guillemin and Sternberg on abstract transitive Lie algebras ([3] and [11]).

$\$ 2$ is devoted to elementary properties of induced and produced modules. We also show how produced Lie algebra modules and induced Lie group modules are related to each other. In $\S 3$ we introduce our main tool, a product structure between members of produced Lie algebra modules. This structure arises from the hyperalgebra structure possessed by universal enveloping algebras (see [10]) and reflects the natural product structure between members of induced Lie group modules. Using our product structure we prove in $\$ 4$ a generalization of the Realization Theorem of Guillemin and Sternberg. The remaining two sections are devoted to proving Lie algebra analogues of theorems of G. W. Mackey ([7] and [8]) concerning systems of imprimitivity and concerning irreducibility criteria for induced representations of group extensions.

The present author wishes to express his thanks to Professors H. Zassenhaus, R. Steinberg, R. Arens, and S. Sternberg for conversations over a period of years bearing on the subject of this paper.

2. Induction and production. Let $A$ be an associative algebra with 1 over a field $K$ and let $B$ be a subalgebra of $A$ containing 1. Following D. G. Higman [6], we make the following definitions:

Received by the editors October 8, 1968.

(1) This research was supported in part by the National Science Foundation (NSF GP-6727). 
Definition. Let $V$ be a unitary $B$-module. A pair $(U, \varphi)$ consisting of a unitary $A$-module $U$ and a $B$-homomorphism $\varphi$ from $V$ into $U$ (resp. from $U$ into $V$ ) is said to be induced (resp. produced) from $V$ if, for every pair $(W, \sigma)$ as above, there exists a unique $A$-homomorphism $\theta$ from $U$ into $W$ (resp. from $W$ into $U$ ) such that

$$
\sigma=\theta \circ \varphi \quad(\text { resp. } \sigma=\varphi \circ \theta) .
$$

Observe that these definitions are simply a formalization of the Frobenius reciprocity law. Indeed, what is required is that the map $\theta \rightarrow \theta \circ \varphi$ of $\operatorname{Hom}_{A}(U, W)$ into $\operatorname{Hom}_{B}(V, W)$ (resp. the map $\theta \rightarrow \varphi \circ \theta$ of $\operatorname{Hom}_{A}(W, U)$ into $\operatorname{Hom}_{B}(W, V)$ ) be bijective.

[6] tells us that induced and produced pairs exist and are unique up to the obvious notion of isomorphism. Indeed, we may construct an induced (resp. produced) pair as follows: The underlying vector space of $U$ is $A \otimes_{B} V$ (resp. $\operatorname{Hom}_{B}(A, V)$ ). Here $A$ is regarded as a right (resp. left) $B$-module. The action of $a \in A$ on $u \in U$ is given by $a u=(a b) \otimes v$ if $u=b \otimes v$ where $b \in A$ and $v \in V$ (resp. $(a u)(b)=u(b a)$ for all $b \in A$ ). And finally $\varphi$ is defined by $\varphi(v)=1 \otimes v$ for all $v \in V$ (resp. $\varphi(u)=u(1)$ for all $u \in U)$. For a pair $(W, \sigma)$ as in the definition, the map $\theta$ is given by $\theta(b \otimes v)=b \sigma(v)$, where $b \in A$ and $v \in V$ (resp. $\theta(w) b=\sigma(b w)$ for all $w \in W$ and $b \in A$ ).

Let $\mathfrak{g}$ be a Lie algebra over $K$ with subalgebra $\mathfrak{h}$. Replacing $A$ by $\mathfrak{g}$ and $B$ by $\mathfrak{h}$ we obtain the notions of induced and produced modules of Lie algebra. Let $\mathfrak{H}(\mathfrak{g})$ (resp. $\mathfrak{u}(\mathfrak{h}))$ be the universal enveloping algebra of $\mathfrak{g}$ (resp. $\mathfrak{h})$. Regard $\mathfrak{g}$ as a subspace of $\mathfrak{U}(\mathfrak{g})$ and identify $\mathfrak{u}(\mathfrak{h})$ with the subalgebra of $\mathfrak{U}(\mathfrak{g})$ generated by $\mathfrak{h}$ and 1 . Because of the correspondence between $\mathfrak{g}$-modules and unitary $\mathfrak{u}(\mathfrak{g})$-modules, we are led from induction and production for the Lie algebra pair $(\mathfrak{g}, \mathfrak{h})$ to induction and production for the associative algebra pair $(\mathfrak{u}(\mathfrak{g}), \mathfrak{u}(\mathfrak{h}))$.

As Higman points out ([6], §2), the notions of induced and produced pairs are in a formal sense dual to each other. In our case, we have more.

Proposition 1. Let $V$ be an $\mathfrak{h}$-module. Let $V^{*}$ be the contragredient $\mathfrak{h}$-module to $V$. Let $(U, \varphi)\left(\right.$ resp. $\left.\left(U^{\prime}, \varphi^{\prime}\right)\right)$ be a pair induced from $V\left(\right.$ resp. produced from $\left.V^{*}\right)$. Then the $\mathrm{g}$-module $U^{\prime}$ is isomorphic to the g-module $U^{*}$ contragredient to $U$.

Recall that for any $\mathrm{g}$-module $W$, the contragredient $\mathrm{g}$-module $W^{*}$ is defined by letting its underlying vector space be the (algebraic) dual of the underlying vector space of $W$ and defining the action of $\mathfrak{g}$ on $W^{*}$ by $(x f)(v)=-f(x v)$ for all $x \in \mathfrak{g}$, $f \in W^{*}, v \in W$. The mapping $x \rightarrow-x$ extends to an antiautomorphism of period two of $\mathfrak{u}(\mathfrak{g})$, called the main antiautomorphism and denoted by '. The restriction of ' to $\mathfrak{u}(\mathfrak{h})$ is the main antiautomorphism of $\mathfrak{u}(\mathfrak{h})$. Observe also that $(a f)(v)=f\left(a^{\prime} v\right)$ for $v \in W, f \in W^{*}$, and $u \in \mathfrak{H}(\mathfrak{g})$.

Proof. Realize $U$ as $\mathfrak{u}(\mathfrak{g}) \otimes \mathfrak{u}_{(\mathfrak{h})} V$ and $U^{\prime}$ as $\operatorname{Hom}_{\mathfrak{u}(\mathfrak{h})}\left(\mathfrak{u}(\mathfrak{g}), V^{*}\right)$. Let $\psi \in$ $\left(\mathfrak{u}(\mathfrak{g}) \otimes \mathfrak{u}_{(\mathfrak{G})} V\right)^{*}$ and let $a \in \mathfrak{u}(\mathfrak{g})$. Define $\hat{\psi}(a)$ by $\hat{\psi}(a)(v)=\psi\left(a^{\prime} \otimes v\right)$ for all $v \in V$. Plainly, $\hat{\psi}(a) \in V^{*}$ and $\hat{\psi} \in \operatorname{Hom}_{K}\left(\mathfrak{u}(\mathfrak{g}), V^{*}\right)$. Let $z \in \mathfrak{u}(\mathfrak{h})$. Then $\hat{\psi}(z a)(v)=\psi\left(a^{\prime} z^{\prime} \otimes v\right)=$ $\psi\left(a^{\prime} \otimes z^{\prime} v\right)=\hat{\psi}(a)\left(z^{\prime} v\right)=(z \hat{\psi}(a))(v)$. Hence $\hat{\psi} \in U^{\prime}$. 
Plainly, the map $\psi \rightarrow \hat{\psi}$ is linear. If $b \in \mathfrak{U}(\mathrm{g})$ and $\psi \in U^{*}$, we have $(b \psi)^{\wedge}(a)(v)$ $=(b \psi)\left(a^{\prime} \otimes v\right)=\psi\left(b^{\prime}\left(a^{\prime} \otimes v\right)\right)=\psi\left((a b)^{\prime} \otimes v\right)=\hat{\psi}(a b)(v)=(b \hat{\psi}(a))(v)$ for all $a \in \mathfrak{U}(\mathrm{g})$ and $v \in V$. Thus $\wedge$ is a $\mathfrak{u}(\mathrm{g})$-homomorphism. Moreover, if $\hat{\psi}=0$, then $\psi$ vanishes on a set of generators for $U$, so that $\psi=0$.

It remains to show that $\widehat{ }$ is surjective. Let $\zeta \in U^{\prime}$. Define $\xi(a, v)$ by $\xi(a, v)$ $=\zeta\left(a^{\prime}\right)(v)$ for all $a \in \mathfrak{u}(\mathfrak{g})$ and $v \in V$. $\xi$ is bilinear on $\mathfrak{u}(\mathfrak{g}) \times V$ and $\xi(a z, v)=\zeta\left(z^{\prime} a^{\prime}\right)(v)$ $=\zeta\left(a^{\prime}\right)(z v)=\xi(a, z v)$ for all $z \in \mathfrak{U}(\mathfrak{h})$. Thus there exists a unique $\psi \in U^{*}$ such that $\psi(a \otimes v)=\xi(a, v)=\zeta\left(a^{\prime}\right)(v)$. Therefore $\hat{\psi}=\zeta$ and our proposition is proved.

COROLlaRY. Let $K$ be a (Hausdorff) topological field. Realize $U$ and $U^{\prime}$ as in the proposition. Give $V^{*}$ and $U^{*}$ the weak topologies they get from the dual pairings $\left(V, V^{*}\right)$ and $\left(U, U^{*}\right)$. Give $U^{\prime}$ the finite-open topology. Then ${ }^{\wedge}$ is a homeomorphism.

Proof. Let $\left\{\psi_{\alpha}\right\}$ be a net in $U^{*}$ and let $\psi \in U^{*}$. Then $\psi_{\alpha} \rightarrow \psi$ if and only if $\psi_{\alpha}(u) \rightarrow \psi(u)$ for all $u \in U$. Since the products $a \otimes v$ with $a \in \mathfrak{u}(\mathrm{g})$ and $v \in V$ generate $U$ additively, we have $\psi_{\alpha} \rightarrow \psi$ if and only if $\hat{\psi}_{\alpha}(a)(v)=\psi_{\alpha}(a \otimes v) \rightarrow \psi(a \otimes v)$ $=\hat{\psi}(a)(v)$. By definition of the topologies on $V^{*}$ and $U^{\prime}$, this holds if and only if $\hat{\psi}_{\alpha}(a) \rightarrow \hat{\psi}(a)$ for all $a$, which holds if and only if $\hat{\psi}_{\alpha} \rightarrow \hat{\psi}$ in $U^{\prime}$.

REMARK 1. There is a bijection (viz., annihilation) between the invariant subspaces of $V$ (resp. $U$ ) and the closed invariant subspaces of $V^{*}$ (resp. $U^{*}$ ). Thus Proposition 1 and its corollary allow us to translate irreducibility criteria for $U$ into topological irreducibility criteria for $U^{\prime}$. We shall make use of this in $\S 6$.

REMARK 2. The most common uses of the proposition and its corollary are:

(a) $K$ is arbitrary and is given the discrete topology.

(b) $K=\boldsymbol{R}$ or $\boldsymbol{C}$ with the usual topology.

Note that if $K=\boldsymbol{R}$ or $\boldsymbol{C}$ then $V^{*}$ (resp. $U^{*}$, resp. $U^{\prime}$ ) has the same set of invariant subspaces no matter whether we use option (a) or option (b).

Let $I$ be a simply ordered index set whose cardinality is $\operatorname{dim}_{K}(\mathfrak{g} / \mathfrak{h})$. Let $\bar{x} \in(\mathfrak{g} / \mathfrak{h})^{I}$ be chosen so that $\left\{\bar{x}_{i}: i \in I\right\}$ is a basis for $\mathfrak{g} / \mathfrak{h}$. For each $i \in I$, choose $x_{i} \in \mathfrak{g}$ such that $x_{i} \in \bar{x}_{i}$. Let $J$ denote the nonnegative integers and let $Z$ denote those $m \in J^{I}$ such that $m_{i}=0$ for all but a finite number of $i \in I .|m|=\sum_{i \in I} m_{i}$. If $m \in Z, x^{m}$ will denote $x_{i_{1}}^{m_{i_{1}}} \cdots x_{i_{n}}^{m_{i_{n}}}$ where $i_{n}>\cdots>i_{1}$ and $\left\{i_{1}, \ldots, i_{n}\right\}=\left\{i: m_{\mathfrak{i}} \neq 0\right\}$, this product being taken in $\mathfrak{u}(\mathfrak{g})$. We may regard $\mathfrak{g} / \mathfrak{h}$ as an abelian Lie algebra, so that $\mathfrak{u}(\mathfrak{g} / \mathfrak{h})=S(\mathfrak{g} / \mathfrak{h})$, the symmetric algebra. Then $\bar{x}^{m}$ will be defined in $S(\mathfrak{g} / \mathfrak{h})$ analagously to $x^{m}$. Let $\tau$ be the linear map of $S(\mathfrak{g} / \mathfrak{h})$ into $\mathfrak{u}(\mathfrak{g})$ which sends $\bar{x}^{m}$ into $x^{m}$.

Let $V$ be an $\mathfrak{h}$-module. We define $\iota: S(\mathfrak{g} / \mathfrak{h}) \otimes_{K} V \rightarrow \mathfrak{u}(\mathfrak{g}) \otimes \mathfrak{u}(\mathfrak{h}) \quad V$ by the formula $\iota(a \otimes v)=\tau(a) \otimes v$ for $a \in S(\mathfrak{g} / \mathfrak{h})$ and $v \in V$. The Poincaré-Birkhoff-Witt theorem implies that $\left\{x^{m}: m \in Z\right\}$ is a basis of the right $\mathfrak{u}(\mathfrak{h})$-module $\mathfrak{u}(\mathfrak{g})$. It follows that $\iota$ is bijective. Similarly, we define $\pi: \operatorname{Hom}_{\mathfrak{u}(\mathfrak{h})}(\mathfrak{u}(\mathfrak{g}), V) \rightarrow \operatorname{Hom}_{K}(S(\mathfrak{g} / \mathfrak{h}), V)$ by the formula $(\pi u)(a)=u(\tau(a))$ for $u \in \operatorname{Hom}_{\mathfrak{U}_{(\mathfrak{h})}}(\mathfrak{u}(\mathfrak{g}), V)$ and $a \in S(\mathfrak{g} / \mathfrak{h})$. Since $\left\{x^{m}: m \in Z\right\}$ is a basis of the left $\mathfrak{u}(\mathfrak{h})$-module $\mathfrak{u}(\mathfrak{g})$, we see that $\pi$ is bijective. Clearly, $\iota$ and $\pi$ depend on the choice of $x$. 
Let $V$ be an $\mathfrak{h}$-module and let $U=\mathfrak{u}(\mathfrak{g}) \otimes \mathfrak{u}_{(\mathfrak{h})} V$. Let $\mathfrak{u}_{p}(\mathfrak{g})$ be the span of all products $y_{1} \cdots y_{q}$ in $\mathfrak{u}(\mathfrak{g})$, where $y_{1}, \ldots, y_{q} \in \mathfrak{g}$ and $0 \leqq q \leqq p$. Set $\mathfrak{u}_{p}(\mathfrak{g})=\{0\}$ for $p \leqq-1$. This filtration on $\mathfrak{u}(\mathfrak{g})$ gives rise to a filtration on $U$ : let $U_{p}$ be the span in $U$ of all elements of the form $a \otimes v$ where $a \in \mathfrak{U}_{p}(\mathfrak{g})$ and $v \in V$. Similarly there is a (downward) filtration on $U^{\prime}=\operatorname{Hom}_{\mathfrak{U}_{(\mathfrak{g})}}(\mathfrak{u}(\mathfrak{g}), V): U_{p}^{\prime}=\left\{u \in U^{\prime}: u \mid \mathfrak{u}_{p-1}(\mathfrak{g})=0\right\}$. We may use the filtration on $U^{\prime}$ to give it the structure of topological vector space, if $K$ is given the discrete topology. The filtration topology on $U^{\prime}$ coincides with the finite-open topology if $V$ is discrete and $\operatorname{dim}(\mathfrak{g} / \mathfrak{h})<\infty$.

Since $S(\mathfrak{g} / \mathfrak{h})=\mathfrak{u}(\mathfrak{g} / \mathfrak{h})$ where $\mathfrak{g} / \mathfrak{h}$ is given the abelian Lie structure, $S(\mathfrak{g} / \mathfrak{h}) \otimes_{K} V$ (resp. $\operatorname{Hom}_{K}(S(\mathfrak{g} / \mathfrak{h}), V)$ ) is the $\mathfrak{g} / \mathfrak{h}$-module induced (resp. produced) from the trivial $\{0\}$-module $V$. Thus we may filter these modules as above. We then obtain

Proposition 2. $\iota$ and $\pi$ are filtration preserving.

Proof. We shall give the proof for $\iota$. The proof for $\pi$ is quite similar. Let $U=\mathfrak{u}(\mathfrak{g}) \otimes \mathfrak{u}_{(\mathfrak{h})} V$ and $\bar{U}=S(\mathfrak{g} / \mathfrak{h}) \otimes_{K} V$. Clearly $\iota\left(\bar{U}_{p}\right) \subseteq U_{p}$. Embed $I$ in a simply ordered set $I^{\prime}$ so that $i \in I$ and $i^{\prime} \in I^{\prime}-I$ imply $i^{\prime}>i$ and so that the cardinality of $I^{\prime}=\operatorname{dim} g$. Choose $x \in \mathfrak{g}^{I^{\prime}}$ so that $\left\{x_{i}: i \in I^{\prime}-I\right\}$ is a basis for $\mathfrak{h}$ and so that $\left\{x_{i}: i \in I\right\}$ is the previously chosen basis of $g$ modulo $\mathfrak{h}$. We define $Z^{\prime}$ to be the set of almost zero $m \in J^{I^{\prime}} \cdot|m|=\sum_{i \in I^{\prime}} m_{i}$. If $m \in Z^{\prime}, x^{m}$ is defined in the obvious way. Now by the Poincaré-Birkhoff-Witt theorem, any product $y_{1} \cdots y_{q}$ in $\mathfrak{u}(\mathfrak{g})$ with $y_{k} \in \mathfrak{g}$ may be written as a linear combination of $x^{m}$ with $m \in Z^{\prime}$ and $|m| \leqq q$. Therefore any $u \in U_{p}$ is a linear combination of elements of the form $x^{m} \otimes v_{m}$ where $|m| \leqq p$ and $v_{m} \in V$. Let $l$ (resp. $r$ ) be defined by $l_{i}=m_{i}$ (resp. $r_{i}=0$ ) if $i \in I$ and $l_{i}=0$ (resp. $r_{i}=m_{i}$ ) if $i \in I^{\prime}-I$. Then $x^{m}=x^{l} x^{r}$ and $x^{r} \in \mathfrak{u}(\mathfrak{h})$. Therefore $x^{m} \otimes v_{m}=x^{l} \otimes x^{r} v_{m} \in \iota\left(\bar{U}_{p}\right)$ because $|l| \leqq|m| \leqq p$.

3. The multiplicative structure of produced representations. Let $V_{1}$ and $V_{2}$ be $\mathfrak{h}$-modules and hence $\mathfrak{u}(\mathfrak{h})$-modules. Then the tensor product of the underlying vector spaces becomes naturally a $\mathfrak{u}(\mathfrak{h}) \otimes \mathfrak{u}(\mathfrak{h})$-module, denoted by $V_{1} \times V_{2}$. $\mathfrak{u}(\mathfrak{h}) \otimes \mathfrak{u}(\mathfrak{h})$ is canonically isomorphic to $\mathfrak{u}(\mathfrak{h} \oplus \mathfrak{h})$, so that we regard $V_{1} \times V_{2}$ as an $\mathfrak{h} \oplus \mathfrak{h}$-module. Thus $\operatorname{Hom}_{\mathfrak{U}(\mathfrak{h} \oplus \mathfrak{h})}\left(\mathfrak{U}(\mathfrak{g} \oplus \mathfrak{g}), V_{1} \times V_{2}\right)$ is a $\mathfrak{g} \oplus \mathfrak{g}$-module. Note that if $u_{1} \in \operatorname{Hom}_{\mathfrak{u}(\mathfrak{g})}\left(\mathfrak{u}(\mathfrak{g}), V_{\mathfrak{i}}\right), i=1,2$, then $u_{1} \times u_{2}$, mapping $\mathfrak{u}(\mathfrak{g}) \otimes \mathfrak{u}(\mathfrak{g})$ into $V_{1} \times V_{2}$, defined by $\left(u_{1} \times u_{2}\right)(a \otimes b)=u_{1}(a) \times u_{2}(b)$ for $a, b \in \mathfrak{H}(g)$ in fact belongs to $\operatorname{Hom}_{\mathfrak{u}(\mathfrak{h} \oplus \mathfrak{h})}\left(\mathfrak{u}(\mathfrak{g} \oplus \mathfrak{g}), V_{1} \times V_{2}\right)$. Moreover, the map $\left(u_{1}, u_{2}\right) \rightarrow u_{1} \times u_{2}$ gives rise to a map of $\operatorname{Hom}_{\mathfrak{U}(\mathfrak{h})}\left(\mathfrak{H}(\mathfrak{g}), V_{1}\right) \times \operatorname{Hom}_{\mathfrak{U}_{(\mathfrak{h})}}\left(\mathfrak{u}(\mathfrak{g}), V_{2}\right)$ into $\operatorname{Hom}_{\mathfrak{U}_{(\mathfrak{h} \oplus \mathfrak{h})}}\left(\mathfrak{U}(\mathfrak{g} \oplus \mathfrak{g}), V_{1} \times V_{2}\right)$. This map is a $\mathfrak{u}(\mathfrak{g}) \otimes \mathfrak{u}(\mathfrak{g})$-homomorphism.

We may also consider the h-module $V_{1} \otimes V_{2}$, whose underlying vector space is that of $V_{1} \times V_{2}$, with the $\mathfrak{h}$-action $h\left(v_{1} \otimes v_{2}\right)=\left(h v_{1}\right) \otimes v_{2}+v_{1} \otimes\left(h v_{2}\right)$. This action may be related to the action of $\mathfrak{u}(\mathfrak{h} \oplus \mathfrak{h})$ on $V_{1} \times V_{2}$ as follows: Let $\Delta$ be the diagonal map of $\mathfrak{g}$ into $\mathfrak{g} \oplus \mathrm{g}$ given by $\Delta(x)=x \oplus x . \Delta$ is a homomorphism and hence extends to a homomorphism of $\mathfrak{u}(\mathfrak{g})$ into $\mathfrak{u}(\mathfrak{g} \oplus \mathfrak{g})$. We have $h\left(v_{1} \otimes v_{2}\right)$ $=\Delta(h)\left(v_{1} \times v_{2}\right)$ for $h \in \mathfrak{h}$, with the obvious abuse of notation. 
Given $u_{i} \in \operatorname{Hom}_{\mathfrak{u}_{(\mathfrak{g})}}\left(\mathfrak{u}(\mathfrak{g}), V_{i}\right), i=1,2$, we define a map $\dot{u}_{1} \otimes u_{2}$ of $\mathfrak{u}(\mathfrak{g})$ into $V_{1} \times V_{2}$ by $\left(u_{1} \otimes u_{2}\right)(a)=\left(u_{1} \times u_{2}\right)(\Delta(a))$. Here we are using the coproduct $\Delta$ in the hyperalgebra $\mathfrak{u}(\mathfrak{g})$ to define a multiplication in certain dual spaces, exactly as in [10].

Lemma 1. $u_{1} \otimes u_{2} \in \operatorname{Hom}_{\mathfrak{U}(\mathfrak{G})}\left(\mathfrak{u}(\mathfrak{g}), V_{1} \otimes V_{2}\right)$.

Proof. Let $a \in \mathfrak{U}(\mathfrak{g}), z \in \mathfrak{U}(\mathfrak{h})$. Then

$$
\begin{aligned}
\left(u_{1} \otimes u_{2}\right)(z a) & =\left(u_{1} \times u_{2}\right)(\Delta(z a))=\left(u_{1} \times u_{2}\right)(\Delta(z) \Delta(a)) \\
& =\Delta(z)\left(\left(u_{1} \times u_{2}\right)(\Delta(a))\right)=z\left(\left(u_{1} \otimes u_{2}\right)(a)\right) .
\end{aligned}
$$

Let $\mathscr{P}(\mathfrak{g}, \mathfrak{h})$ denote the class of all $\mathfrak{g}$-modules $\operatorname{Hom}_{\mathfrak{U}(\mathfrak{h})}(\mathfrak{u}(\mathfrak{g}), V)$, where $V$ runs over all $\mathfrak{h}$-modules. We have defined a multiplication on $\bigcup \mathscr{P}(\mathfrak{g}, \mathfrak{h})$, which is clearly bilinear. Moreover, it is associative: if $\sigma$ is the canonical isomorphism of $\left(V_{1} \otimes V_{2}\right) \otimes V_{3}$ onto $V_{1} \otimes\left(V_{2} \otimes V_{3}\right)$ and if $u_{i} \in \operatorname{Hom}_{\mathfrak{U}_{(\mathfrak{G})}}\left(\mathfrak{u}(\mathfrak{g}), V_{i}\right), i=1,2,3$, then $\sigma \circ\left(\left(u_{1} \otimes u_{2}\right) \otimes u_{3}\right)=u_{1} \otimes\left(u_{2} \otimes u_{3}\right)$.

Proposition 3. Each $x \in \mathfrak{g}$ acts on $\bigcup \mathscr{P}(\mathfrak{g}, \mathfrak{h})$ as a derivation; i.e. if $u_{\mathfrak{i}} \in U_{\mathfrak{i}}^{\prime} \in$ $\mathscr{P}(\mathfrak{g}, \mathfrak{h}), i=1,2$, then $x\left(u_{1} \otimes u_{2}\right)=\left(x u_{1}\right) \otimes u_{2}+u_{1} \otimes\left(x u_{2}\right)$.

Proof. Let $a \in \mathfrak{U}(\mathfrak{g})$. Then

$$
\begin{aligned}
{\left[x\left(u_{1} \otimes u_{2}\right)\right](a) } & =\left(u_{1} \otimes u_{2}\right)(a x)=\left(u_{1} \times u_{2}\right)(\Delta(a x)) \\
& =\left(u_{1} \times u_{2}\right)(\Delta(a) \Delta(x))=\left[\Delta(x)\left(u_{1} \times u_{2}\right)\right](\Delta(a)) \\
& =\left[\left(x u_{1}\right) \times u_{2}+u_{1} \times\left(x u_{2}\right)\right](\Delta(a)) \\
& =\left[\left(x u_{1}\right) \otimes u_{2}+u_{1} \otimes\left(x u_{2}\right)\right](a) .
\end{aligned}
$$

Next we relate these operations to the filtrations introduced in $\$ 2$ on produced modules.

Lemma 2. Let $y_{1}, \ldots, y_{p} \in \mathfrak{g}$. Then

$$
\Delta\left(y_{1}^{m_{1}} \cdots y_{p}^{m_{p}}\right)=\sum_{0 \leqq k_{i} \leqq m_{i}}\left(\begin{array}{l}
m_{1} \\
k_{1}
\end{array}\right) \cdots\left(\begin{array}{l}
m_{p} \\
k_{p}
\end{array}\right) y_{1}^{k_{1}} \cdots y_{p}^{k_{p}} \otimes y_{1}^{m_{1}-k_{1}} \cdots y_{p}^{m_{p}-k_{p}}
$$

in $\mathfrak{u}(\mathfrak{g})$.

Proof. If $m_{1}=\cdots=m_{p}=1$, then $\Delta\left(y_{1} \cdots y_{p}\right)=\left(y_{1} \otimes 1+1 \otimes y_{1}\right) \cdots\left(y_{p} \otimes 1+\right.$ $\left.1 \otimes y_{p}\right)$ which multiplies out to give our formula. The general case of the lemma follows upon applying this result to $z_{1} \cdots z_{m}, m=\sum m_{i}$, where $z_{1}=\cdots=z_{m_{1}}=y_{1}$, $z_{m_{1}+1}=\cdots=z_{m_{1}+m_{2}}=y_{2}$, etc., and collecting terms.

Corollary.

$$
\Delta\left(U_{p}(\mathfrak{g})\right) \subseteq \sum_{k}^{p} \mathfrak{u}_{k}(\mathfrak{g}) \otimes \mathfrak{u}_{p-k}(\mathfrak{g})
$$

Proposition 4. Let $U_{i}=\operatorname{Hom}_{\mathfrak{U}(\mathfrak{g})}\left(\mathfrak{U}(\mathfrak{g}), V_{i}\right), i=1,2$. Let

$$
U_{3}=\operatorname{Hom}_{\mathfrak{U}_{(\mathfrak{h} \oplus \mathfrak{h})}}\left(\mathfrak{u}(\mathfrak{g} \oplus \mathfrak{g}), V_{1} \times V_{2}\right) \quad \text { and } \quad U_{4}=\operatorname{Hom}_{\mathfrak{u}(\mathfrak{g})}\left(\mathfrak{u}(\mathfrak{g}), V_{1} \otimes V_{2}\right) \text {. }
$$


Suppose $u_{1} \in\left(U_{1}\right)_{p}$ and $u_{2} \in\left(U_{2}\right)_{q}$. Then $u_{1} \times u_{2} \in\left(U_{3}\right)_{p+q}, u_{1} \otimes u_{2} \in\left(U_{4}\right)_{p+q}$, and $x u_{1} \in\left(U_{1}\right)_{p-1}$ for all $x \in \mathfrak{g}$.

Proof. Identify $\mathfrak{u}(\mathfrak{g} \oplus \mathfrak{g})$ with $\mathfrak{u}(\mathfrak{g}) \otimes \mathfrak{u}(\mathfrak{g})$. Then

$$
\mathfrak{u}_{m}(\mathfrak{g} \oplus \mathfrak{g})=\sum_{k} 0^{m} \mathfrak{u}_{k}(\mathfrak{g}) \otimes \mathfrak{u}_{m-k}(\mathfrak{g})
$$

This gives the result for $u_{1} \times u_{2}$. The corollary of Lemma 2 gives the result for $u_{1} \otimes u_{2}$. Finally if $a \in \mathfrak{u}_{p-2}(g)$, then $a x \in \mathfrak{H}_{p-1}(g)$, whence $\left(x u_{1}\right)(a)=u_{1}(a x)=0$ and $x u_{1} \in\left(U_{1}\right)_{p-1}$.

The notion of produced representation of Lie algebras is intimately connected with the conventional notion of induced representation of Lie groups. Let $G$ be a connected real Lie group with closed connected subgroup $H$. Let $L$ be a continuous representation of $H$ on the real finite dimensional vector space $V$. Let $C_{H}^{\infty}(G, V)$ be the space of all $C^{\infty}$ functions $g$ from $G$ to $V$ such that $g(\xi \alpha)=L_{\xi} f(\alpha)$ for all $\xi \in H$ and $\alpha \in G$. $G$ acts upon $C_{H}^{\infty}(G, V)$ according to the formula $(\beta g)(\alpha)=g(\alpha \beta)$. For each $g \in C_{H}^{\infty}(G, V)$, we define $\tilde{g}: \mathfrak{u}(\mathfrak{g}) \rightarrow V$ by $\tilde{g}(a)=(a g)(\varepsilon)$. Here $\mathfrak{u}(\mathfrak{g})$ is realized in the usual way as the algebra of all left invariant differential operators on $G$. (In particular, $(x g)(\alpha)=\left.D_{t} g(\alpha \exp t x)\right|_{t=0}$ for $x \in \mathrm{g}$.) $\varepsilon$ is the identity element of $G$.

We turn $V$ into an $\mathfrak{h}$-module by setting $h v=\left.D_{t} L_{\exp t h} v\right|_{t=0}$. If $g \in C_{H}^{\infty}(G, V)$ and $a \in \mathfrak{H}(\mathrm{g})$, then $a g \in C_{H}^{\infty}(G, V)$. Thus $C_{H}^{\infty}(G, V)$ is a $\mathfrak{u}(\mathrm{g})$-module. We now have

Proposition 5. is a $\mathfrak{U}(\mathfrak{g})$-homomorphism of $C_{H}^{\infty}(G, V)$ into $\operatorname{Hom}_{\mathfrak{U}_{(\mathfrak{h})}}(\mathfrak{U}(\mathfrak{g}), V)$.

Proof. Let $h \in \mathfrak{h}, a \in \mathfrak{u}(\mathfrak{g})$, and $g \in C_{H}^{\infty}(G, V)$. Then

$$
\begin{aligned}
\tilde{g}(h a)=(h a g)(\varepsilon) & =\left.D_{t}(a g)(\exp t h)\right|_{t=0} \\
& =\left.D_{t} L_{\exp t h}(a g)(\varepsilon)\right|_{t=0}=h \tilde{g}(a) .
\end{aligned}
$$

Thus $\tilde{g} \in \operatorname{Hom}_{\mathfrak{U}_{(\mathfrak{h})}}(\mathfrak{u}(\mathfrak{g}), V)$. Now suppose $x \in \mathfrak{g}$. Then $(x g)^{\sim}(a)=(\operatorname{axg})(\varepsilon)=\tilde{g}(a x)$ $=(x \tilde{g})(a)$. Thus $\sim$ is a $\mathfrak{u}(\mathfrak{g})$-homomorphism.

This shows that the $\mathfrak{H}(\mathfrak{g})$-module structure on $\operatorname{Hom}_{\mathfrak{U}(\mathfrak{h})}(\mathfrak{u}(\mathfrak{g}), V)$ is a reflection of the $\mathfrak{u}(\mathrm{g})$-module structure on $C_{H}^{\infty}(G, V)$. One can even show that $\sim$ is surjective.

We finish this section by showing that $\mathbf{X}$ (resp. $\otimes$ ) is a reflection of the natural $\mathbf{X}$ (resp. $\otimes$ ) defined for induced group representations. If $g_{i} \in C_{H}^{\infty}\left(G, V_{i}\right), i=1,2$, we define $g_{1} \times g_{2}: G \times G \rightarrow V_{1} \times V_{2}$ by $\left(g_{1} \times g_{2}\right)\left(\alpha_{1}, \alpha_{2}\right)=g_{1}\left(\alpha_{1}\right) \times g_{2}\left(\alpha_{2}\right) . L_{1} \times L_{2}$ is the representation of $H \times H$ on $V_{1} \times V_{2}$ given by

$$
\left(L_{1} \times L_{2}\right)_{\left(\xi_{1}, \xi_{2}\right)}\left(v_{1} \times v_{2}\right)=\left(L_{1 \xi_{1}} v_{1}\right) \times\left(L_{2 \xi_{2}} v_{2}\right)
$$

Then $g_{1} \times g_{2} \in C_{H \times H}^{\infty}\left(G \times G, V_{1} \times V_{2}\right)$. Observe that the $\mathfrak{u}(\mathfrak{h} \oplus \mathfrak{h})$-module structure on $V_{1} \times V_{2}$ determined by $L_{1} \times L_{2}$ is the $\times$-product of the $\mathfrak{u}(\mathfrak{h})$-module structures on $V_{1}$ and $V_{2}$.

We define similarly $g_{1} \otimes g_{2}: G \rightarrow V_{1} \otimes V_{2}$ by $\left(g_{1} \otimes g_{2}\right)(\alpha)=g_{1}(\alpha) \otimes g_{2}(\alpha)$ and $L_{1} \otimes L_{2}$ by $\left(L_{1} \otimes L_{2}\right)_{\xi}\left(v_{1} \otimes v_{2}\right)=\left(L_{1 \xi} v_{1}\right) \otimes\left(L_{2 \xi} v_{2}\right)$. Then $g_{1} \otimes g_{2} \in C_{H}^{\infty}\left(G, V_{1} \otimes V_{2}\right)$. 
We observe that the $\mathfrak{u}(\mathfrak{h})$-module structure on $V_{1} \otimes V_{2}^{\prime}$ determined by $L_{1} \otimes L_{2}$ is the $\otimes$-product of the $\mathfrak{u}(\mathfrak{h})$-module structure on $V_{1}$ and $V_{2}$.

PROPOSITION 6. $\left(g_{1} \times g_{2}\right)^{\sim}=\tilde{g}_{1} \times \tilde{g}_{2}$ and $\left(g_{1} \otimes g_{2}\right)^{\sim}=\tilde{g}_{1} \otimes \tilde{g}_{2}$.

Proof. $\mathfrak{u}(\mathfrak{g}) \otimes \mathfrak{u}(\mathfrak{g})$ is the algebra of all left invariant differential operators on $G \times G$ via the natural isomorphism with $\mathfrak{u}(\mathfrak{g} \oplus \mathfrak{g})$. This correspondence makes $a \otimes b \in \mathfrak{u}(\mathfrak{g}) \otimes \mathfrak{u}(\mathfrak{g})$ operate on a function on $G \times G$ be letting $a$ operate on the first variable and $b$ operate on the second. Therefore

$$
\begin{aligned}
\left(g_{1} \times g_{2}\right)^{\sim}(a \otimes b) & =\left[(a \otimes b)\left(g_{1} \times g_{2}\right)\right](\varepsilon, \varepsilon)=\left[a g_{1} \times b g_{2}\right](\varepsilon, \varepsilon) \\
& =\left(a g_{1}\right)(\varepsilon) \times\left(a g_{2}\right)(\varepsilon)=\tilde{g}_{1}(a) \times \tilde{g}_{2}(b)=\left(\tilde{g}_{1} \times \tilde{g}_{2}\right)(a \otimes b) .
\end{aligned}
$$

As for $\otimes$, we note that the Leibniz product rule comes down to $\left[a\left(g_{1} \otimes g_{2}\right)\right](\alpha)$ $=\left[\Delta(a)\left(g_{1} \times g_{2}\right)\right](\alpha, \alpha)$. Therefore

$$
\begin{aligned}
\left(g_{1} \otimes g_{2}\right)^{\sim}(a) & =\left[a\left(g_{1} \otimes g_{2}\right)\right](\varepsilon)=\left[\Delta(a)\left(g_{1} \times g_{2}\right)\right](\varepsilon, \varepsilon) \\
& =\left(g_{1} \times g_{2}\right)^{\sim}(\Delta(a))=\left(\tilde{g}_{1} \times \tilde{g}_{2}\right)(\Delta(a))=\left(\tilde{g}_{1} \otimes \tilde{g}_{2}\right)(a) .
\end{aligned}
$$

Remark. Note that $C_{H}^{\infty}(G, V)$ may be regarded as the space of $C^{\infty}$-crosssections of the homogeneous vector bundle over $H \backslash G$ associated to the principal bundle $G \rightarrow H \backslash G$ by the action of $H$ on $V$ given by $L$. Thus $\operatorname{Hom}_{\mathfrak{U}_{(\mathfrak{G})}}(\mathfrak{u}(\mathfrak{g}), V)$ may be thought of as an infinitesimal homogeneous vector bundle. From this point of view $u_{1} \otimes u_{2}$ is the usual tensor product of cross-sections.

Notation. For the rest of this paper $u_{1} \otimes u_{2}$ will be denoted by $u_{1} u_{2}$ whenever $u_{1} \in F=\operatorname{Hom}_{\mathfrak{U}_{(\mathfrak{h})}}(\mathfrak{U}(\mathfrak{g}), K)$ where $K$ is the one-dimensional trivial $\mathfrak{h}$-module. As usual $K \otimes_{K} V$ is identified with $V$. This makes $F$ an associative algebra with identity $e(e(1)=1$ and $e(\mathfrak{g} \mathfrak{u}(\mathfrak{g}))=0)$ and turns every produced module into a unitary $F$ module. This is immediate from Lemma 2.

4. The Guillemin-Sternberg-Rim realization theorem. Let $\mathrm{g}$ be a Lie algebra over a field $K$. Let $\mathfrak{h}$ be a subalgebra of $\mathfrak{g}$. Set $\mathfrak{g}_{p}=\mathfrak{g}$ for $p \leqq-1$ and $\mathfrak{g}_{0}=\mathfrak{h}$. Define $\mathfrak{g}_{p}$ inductively by setting $\mathfrak{g}_{p}=\left\{x \in \mathfrak{g}_{p-1}:[y, x] \in \mathfrak{g}_{p-1}\right.$ for all $\left.y \in \mathfrak{g}\right\}, p \geqq 1$. An easy induction (cf. [3], §6) shows that $\left\{\mathfrak{g}_{p}\right\}$ is a filtered Lie algebra. It is 0 -acyclic (see [9]); if it were complete and if $\operatorname{dim}(\mathfrak{g} / \mathfrak{h})<\infty$, it would be a transitive Lie algebra. The filtration is separated (i.e. $\cap \mathfrak{g}_{p}=\{0\}$ ) if and only if $\mathfrak{h}$ contains no $\mathfrak{g}$-ideals, a property we assume through the end of Theorem 1.

Let $F=\operatorname{Hom}_{\mathfrak{U}_{(\mathfrak{h})}}(\mathfrak{u}(\mathfrak{g}), K)$ be given its filtration (see $\left.\S 2\right)$. Let $D_{p}$ be the space of derivations $d$ of $F$ such that $d F_{m} \subseteq F_{m+p}$ for all $m$ and let $D=\bigcup D_{p} .\left\{D_{p}\right\}$ is a filtered Lie algebra. If $L$ is a Lie subalgebra of $\mathrm{D}$, we filter $L$ by setting $L_{p}=L \cap D_{p}$. For $x \in \mathfrak{g}$, define $\gamma(x) \in \operatorname{Hom}_{K}(F, F)$ by $\gamma(x) u=x u$ for $u \in F$ (i.e. $\gamma$ is the representation of $g$ determined by the $g$-module $F$ ). Finally, note that $F=\operatorname{Ke}+F_{1}$ and that $D_{0} F \subseteq F_{1}$.

THEOREM 1. $\gamma$ is a filtered Lie algebra isomorphism of $\mathrm{g}$ with a subalgebra of $D$. For every Lie algebra homomorphism $\gamma_{1}$ of $\mathfrak{g}$ into $D$ such that $\gamma_{1}(x)-\gamma(x) \in D_{0}$ for all $x \in \mathfrak{g}$, there is a unique filtered algebra automorphism $\theta$ of $F$ such that $\theta \circ \gamma_{1}(x)$ $=\gamma(x) \circ \theta$ for all $x \in \mathfrak{g}$. 
Proof. According to Proposition 3, $\gamma(x)$ is a derivation of $F$. Plainly $\gamma(x) \in D_{-1}$. We show that $\gamma(h) \in D_{0}$ if and only if $h \in \mathfrak{h}$. Suppose $h \in \mathfrak{h}$ and $u \in F_{m}$. Let $a \in \mathfrak{U}_{m-1}(\mathfrak{g})$. Then $(\gamma(h) u)(a)=u(a h)$. But $a h=h a+b$ where $b \in \mathfrak{H}_{m-1}(\mathfrak{g})$. Since $u(h a)=0$ and $u(b)=0, \gamma(h) u \in F_{m}$ and $\gamma(h) \in D_{0}$. Now suppose $h \notin \mathfrak{h}$. Choose $\bar{h} \in \mathfrak{g} / \mathfrak{h} \subseteq S(\mathfrak{g} / \mathfrak{h})$ such that $h \in \bar{h} . \bar{h} \neq 0$. Let $\tau$ and $\pi$ be as in $\S 2$. Then $\tau(\bar{h})=h$. Choose $w \in \operatorname{Hom}_{K}(S(\mathfrak{g} / \mathfrak{h}), K)$ so that $w(\bar{h}) \neq 0$. Set $u=\pi^{-1}(w)$. Then $[\gamma(h) u](1)=u(h)=$ $u(\tau(\bar{h}))=w(\bar{h}) \neq 0$ so that $\gamma(h) u \notin F_{1}$. Therefore $\gamma(x) \notin D_{0}$.

It follows that $\gamma$ is injective. Indeed, let $\mathfrak{t}=\operatorname{Ker} \gamma . x \in \mathfrak{t} \Rightarrow \gamma(x) F_{m}=\{0\} \subseteq F_{m}$ so that $\gamma(x) \in D_{0}$ and hence $x \in \mathfrak{h}$. But $\mathfrak{h}$ contains no $\mathfrak{g}$-ideals. Therefore $\mathfrak{t}=\{0\}$ and $\gamma$ is injective.

We next show that $\gamma\left(\mathrm{g}_{p}\right)=\gamma(\mathrm{g}) \cap D_{p}$. We have already shown this for $p \leqq 0$. Suppose inductively that $\gamma\left(\mathfrak{g}_{p}\right)=\gamma(\mathfrak{g}) \cap D_{p}$ for some $p \geqq 0$. We show that $\gamma\left(\mathfrak{g}_{p+1}\right)$ $=\gamma(\mathrm{g}) \cap D_{p+1}$. Let $x \in \mathrm{g}_{p+1}$. Then $x,[y, x] \in \mathrm{g}_{p}$ for all $y \in g$ so that $\gamma(x)$, $\gamma([y, x]) \in D_{p}$. As a secondary induction, assume that $\gamma(x) F_{m-1} \subseteq F_{m+p}$ for some $m$. We show that $\gamma(x) F_{m} \subseteq F_{m+p+1}$. Let $u \in F_{m}, a \in \mathfrak{u}_{m+p-1}(\mathfrak{g})$, and $y \in \mathfrak{g}$. Then $[\gamma(x) u](a y)=u(a y x)=u(a[y, x])+u(a x y)=[\gamma([y, x]) u](a)+[\gamma(x) \gamma(y) u](a)$. Now

$$
\gamma([y, x]) \in D_{p}
$$

so that $\gamma([y, x]) u \in F_{m+p}$ and the first term vanishes. Again $\gamma(y) u \in F_{m-1}$ and $\gamma(x) F_{m-1} \subseteq F_{m+p}$ so that the second term vanishes. Thus $\gamma(x) u$ vanishes on $\mathfrak{u}_{m+p}(\mathfrak{g})$ and $\gamma(x) u \in F_{m+p+1}$. The secondary induction proves that $\gamma\left(g_{p}\right) \subseteq D_{p+1}$. Conversely, suppose $\gamma(x) \in D_{p+1}$. Let $u \in F_{m}$ and $y \in g$. Then $\gamma([y, x]) u=\gamma(y) \gamma(x) u-\gamma(x) \gamma(y) u$. Since $\gamma(y) \in D_{-1}$ and $\gamma(x) \in D_{p+1}$, the right-hand side belongs to $F_{m+p}$. Thus $\gamma([y, x]) \in D_{p}$. By our induction hypothesis $x,[y, x] \in \mathfrak{g}_{p}$. Therefore $x \in \mathfrak{g}_{p+1}$.

Let $\gamma_{1}$ be as in the statement of the theorem. Note that $\gamma_{1}(x)=\gamma(x)$ (modulo $D_{0}$ ) implies $\gamma_{1}(\mathfrak{g}) \subseteq D_{-1}$ and $\gamma_{1}(\mathfrak{h}) \subseteq D_{0}$. Define $\sigma: F \rightarrow K$ by $\sigma u=u(1)$. Then $\sigma \circ \gamma(h)$ $=\sigma \circ \gamma_{1}(h)=0$ for $h \in \mathfrak{h}$. Since $F$ is produced from the trivial $\mathfrak{h}$-module $K, \S 2$ provides us with a unique $\theta \in \operatorname{Hom}_{K}(F, F)$ such that $\theta \circ \gamma_{1}(x)=\gamma(x) \circ \theta$ for all $x \in \mathfrak{g}$. Indeed, $(\theta u)(a)=\sigma\left(\gamma_{1}(a) u\right)=\left(\gamma_{1}(a) u\right)(1)$ for all $u \in F$ and $a \in \mathfrak{u}(\mathfrak{g})$. (Here $\gamma_{1}$ has been extended to a homomorphism of $\mathfrak{u}(\mathfrak{g})$ into $\operatorname{Hom}_{K}(F, F)$.) Since $\gamma_{1}$ maps $\mathfrak{g}$ into $D_{-1}$, $\gamma_{1}(x) F_{m} \subseteq F_{m-1}$ for all $m$. Therefore $\gamma_{1}\left(\mathfrak{u}_{p}(\mathfrak{g})\right) F_{m} \subseteq F_{m-p}$ for all $m$ and $p$. It follows that $\theta F_{m} \subseteq F_{m}$ so that $\theta$ is filtration preserving.

$\theta$ is a bijection. In fact, let $u \in F_{m}$ and $y \in \mathrm{g}$. We have $\gamma_{1}(y) u=\gamma(y) u$ (modulo $F_{m}$ ). Since $\theta \circ \gamma_{1}(y)=\gamma(y) \circ \theta$ and $\theta$ is filtration preserving, we obtain $\gamma(y) \theta u=\theta \gamma(y) u$ (modulo $F_{m}$ ). If $y_{1}, \ldots, y_{p} \in \mathfrak{g}$, we obtain by iteration $\gamma\left(y_{1} \cdots y_{p}\right) \theta u=\theta \gamma\left(y_{1} \cdots y_{p}\right) u$ (modulo $\left.F_{m-p+1}\right)$. Therefore, if $a \in \mathfrak{u}_{m}(\mathfrak{g}), \quad(\theta u)(a)=[\gamma(a) \theta u](1)=[\theta \gamma(a) u](1)=$ $[\gamma(a) u](1)=u(a)$ because $\theta e=e, \theta F_{1} \subseteq F_{1}$, and $F=K e+F_{1}(e$ is the identity of $F)$. We have shown that $\theta u=u$ (modulo $F_{m}$ ) for all $u \in F_{m}$. It follows that $\theta$ is injective and, since $F$ is filtration complete, that $\theta$ is surjective.

We now show that $\theta\left(u_{1} u_{2}\right)=\left(\theta u_{1}\right)\left(\theta u_{2}\right)$. To see this, we first note that $F$ is a $D$ module, hence a $\mathfrak{u}(D)$-module, so that $F \times F$ is a $\mathfrak{u}(D) \otimes \mathfrak{u}(D)$-module and $F \otimes F$ is a $\mathfrak{u}(D)$-module in the usual ways. The multiplication in $F$ gives rise to a linear 
map $\nu: F \otimes F \rightarrow F$. That $D$ consists of derivations is precisely equivalent to $\nu$ being a $D$-homomorphism, hence a $\mathfrak{u}(D)$-homomorphism. Put in terms of $\nu$ mapping $F \times F \rightarrow F, v$ intertwines the action of $\Delta l$ on $F \times F$ with the action of $l$ on $F$, for all $l \in \mathfrak{U}(D) . \gamma$ and $\gamma_{1}$ are homomorphisms of $\mathfrak{g}$ into $D$ and hence extend to homomorphisms of $\mathfrak{u}(\mathfrak{g})$ into $\mathfrak{u}(D), \gamma \times \mathbf{X} \gamma$ and $\gamma_{1} \times \gamma_{1}$ are homomorphisms of $\mathfrak{u}(\mathfrak{g}) \otimes \mathfrak{u}(\mathfrak{g})$ into $\mathfrak{u}(D) \otimes \mathfrak{u}(D)$. We have $\Delta \circ \gamma=(\gamma \times \gamma) \circ \Delta$ and $\Delta \circ \gamma_{1}=\left(\gamma_{1} \times \gamma_{1}\right) \circ \Delta$. $\theta$ intertwines the representation $\gamma_{1}$ with the representation $\gamma ; \theta \times \theta$ intertwines $\gamma_{1} \times \gamma_{1}$ with $\gamma \times \mathbf{X}$. Let $\sigma$ be as in the previous paragraph. $\sigma \times \boldsymbol{\sigma}$ maps $F \times F$ into $K$. One verifies immediately that $\sigma \circ \gamma_{1}(a)=\sigma \circ \theta \circ \gamma_{1}(a)$ for $a \in \mathfrak{U}(\mathfrak{g})$ and $\sigma \circ \nu=\sigma \times \sigma$. Let $a \in \mathfrak{U}(\mathfrak{g})$. Then

$$
\begin{aligned}
\sigma \circ \gamma(a) \circ \theta \circ \nu & =\sigma \circ \theta \circ \gamma_{1}(a) \circ \nu=\sigma \circ \gamma_{1}(a) \circ \nu \\
& =\sigma \circ \nu \circ\left[\left(\gamma_{1} \times \gamma_{1}\right)(\Delta a)\right]=(\sigma \times \sigma) \circ\left[\left(\gamma_{1} \times \gamma_{1}\right)(\Delta a)\right] \\
& =(\sigma \times \sigma) \circ(\theta \times \theta) \circ\left[\left(\gamma_{1} \times \gamma_{1}\right)(\Delta a)\right] \\
& =(\sigma \times \sigma) \circ[(\gamma \times \gamma)(\Delta a)] \circ(\theta \times \theta) \\
& =\sigma \circ \nu \circ[(\gamma \times \gamma)(\Delta a)] \circ(\theta \times \theta) \\
& =\sigma \circ \gamma(a) \circ \nu \circ(\theta \times \theta) .
\end{aligned}
$$

This implies $\theta \circ \nu=\nu \circ(\theta \times \theta)$; i.e. $\theta$ is multiplicative.

Guillemin and Sternberg, as part of their study of the infinite groups of Cartan, proved a Realization Theorem: every abstract transitive Lie algebra $g$ over a field $K$ of characteristic 0 could be embedded in an essentially unique way in the Lie algebra $D(\mathfrak{g} / \mathfrak{h})$ of continuous derivations of $K\left[\left[(\mathfrak{g} / \mathfrak{h})^{*}\right]\right]$, where $\mathfrak{h}$ is a fundamental subalgebra of $g$ (see $[3, \S 6]$ for definitions and statements, especially Theorem III; also [11, Theorem 4.3]). Rim [9, Corollary 3.9] extended the theorem to $K$ of arbitrary characteristic by redefinining $D(\mathfrak{g} / \mathfrak{h})$ to be a certain subalgebra of the derivations of $S(\mathfrak{g} / \mathfrak{h}) *$ (see $\operatorname{Rim}[9$, p. 356]). Both the Guillemin-Sternberg proof and the Rim proof are cohomological in nature. Both proofs give a noncanonical embedding. Both proofs assume that $\operatorname{dim}(\mathfrak{g} / \mathfrak{h})<\infty$ although this restriction is not essential if things are properly done (see Remark 2 below). Our Theorem 1 embeds $g$ in a filtered Lie algebra $D$ of derivations of $F$. This embedding is canonical and any other embedding which is "close to" it is equivalent to it. There is no restriction on $\operatorname{dim}(\mathfrak{g} / \mathfrak{h})$. There is no cohomology.

We can retrieve the Guillemin-Sternberg-Rim Theorem as follows:

Proposition 7. Let $u_{i} \in \operatorname{Hom}_{\mathfrak{u}_{(\mathfrak{h})}}\left(\mathfrak{U}(\mathfrak{g}), V_{i}\right), i=1,2$. Then

$$
\pi\left(u_{1} \otimes u_{2}\right)=\pi\left(u_{1}\right) \otimes \pi\left(u_{2}\right)
$$

Proof. Let $I$ be an ordered index set for $\mathfrak{g} / \mathfrak{h}$, choose $\bar{x} \in(\mathfrak{g} / \mathfrak{h})^{I}$ and $x \in \mathfrak{g}^{I}$, define $Z \subseteq J^{I}$, and define $\bar{x}^{m}$ and $x^{m}$ for $m \in Z$ as in $\S 2$. The map $\tau: S(\mathfrak{g} / \mathfrak{h}) \rightarrow \mathfrak{u}(\mathfrak{g})$ is defined by $\tau\left(\bar{x}^{m}\right)=x^{m}$. Applying Lemma 2 to $\bar{x}^{m}$ and $x^{m}$, we obtain that

$$
\Delta\left(\tau\left(\bar{x}^{m}\right)\right)=(\tau \otimes \tau)\left(\Delta \bar{x}^{m}\right) ; \quad \text { i.e. } \Delta \circ \tau=(\tau \otimes \tau) \circ \Delta .
$$

This dualizes to give our proposition. 
CoROllary. $F=\operatorname{Hom}_{\mathfrak{U}_{(\mathfrak{h})}}(\mathfrak{u}(\mathfrak{g}), K)$ is isomorphic to the filtered algebra $S(\mathfrak{g} / \mathfrak{h})^{*}$. If char $K=0, F$ is isomorphic to the algebra $K\left[\left[\bar{x}_{i}\right]\right]_{i \in I}$ of formal series in the variables $\left\{\bar{x}_{i}\right\}_{i \in I}$ with coefficients in $K$, filtered by total degree.

Proof. See Rim (loc. cit.) and Cartier [10, Exposé 3, Proposition 6].

Thus we may transfer the action of $\gamma$ to $E=S(\mathfrak{g} / \mathfrak{h}) *$ via $\pi . d \in D_{p}(F)$ if and only if $\pi \circ d \circ \pi^{-1} \in D_{p}(E)$. Thus we obtain a filtered Lie algebra isomorphism $\bar{\gamma}(\cdot)$ $=\pi \circ \gamma(\cdot) \circ \pi^{-1}$ of $\mathrm{g}$ into $D(E)$. In this setting, the restriction $\left(\gamma_{1}-\gamma\right)(\mathrm{g}) \subseteq D_{0}$ has a more natural form. $E$ is a $\mathfrak{g} / \mathfrak{h}$-module $(\mathfrak{g} / \mathfrak{h}$ is abelian!). Let $\delta$ be the corresponding representation. For $y \in \mathfrak{g}$, let $\bar{y}$ be its coset in $\mathfrak{g} / \mathfrak{h}$.

Proposition 8. $\bar{\gamma}(y)-\delta(\bar{y}) \in D_{0}(E)$ for all $y \in \mathfrak{g}$.

Proof. Choose $\left\{x_{i}\right\}_{i \in I}$ as in Proposition 7. Let $i \in I$ and define $r \in Z$ by $\mathrm{r}_{j}=\delta_{i j}$. Let $u \in E_{k}$ and $w=\pi^{-1}(u)$. If $m \in Z$ satisfies $|m| \leqq k-1$, then $\left[\bar{\gamma}\left(x_{i}\right) u\right]\left(\bar{x}^{m}\right)=\left(x_{i} w\right)\left(x^{m}\right)$ $=w\left(x^{m} x_{i}\right)$, while $\left[\delta\left(\bar{x}_{i}\right) u\right]\left(\bar{x}^{m}\right)=u\left(\bar{x}^{m+r}\right)=w\left(x^{m+r}\right)$. By the Poincaré-Birkhoff-Witt Theorem, $x^{m} x_{\mathfrak{i}}=x^{m+r}$ (modulo $\mathfrak{u}_{|m|}(\mathfrak{g})$ ). It follows that $\left[\bar{\gamma}\left(x_{i}\right) u\right]\left(\bar{x}^{m}\right)=\left[\delta\left(\bar{x}_{i}\right) u\right]\left(\bar{x}^{m}\right)$. Thus $\bar{\gamma}\left(x_{i}\right)-\delta\left(\bar{x}_{i}\right) \in D_{0}(E)$. Also $\bar{\gamma}(h)-\delta(\bar{h})=\bar{\gamma}(h) \in D_{0}(E)$ for all $h \in \mathfrak{h}$. Therefore $\bar{\gamma}(y)-\delta(\bar{y}) \in D_{0}(E)$ for all $y \in \mathfrak{g}$.

COROLlary (GuIllemin-STERnBerg-Rim). There exist Lie algebra homomorphisms $\beta$ of $\mathfrak{g}$ into $D(E)$ satisfying $\beta(y)-\delta(\bar{y}) \in D_{0}(E)$ for all $y \in \mathfrak{g}$. Any two such are equivalent under a filtered algebra automorphism of $E$ and are necessarily filtered Lie algebra isomorphisms.

REMARKS (1) The noncanonicalness of the embedding in $D(E)$ shows up in our proof as the noncanonicalness of $\pi$.

(2) One can rescue the Guillemin-Sternberg proof for arbitrary characteristic and arbitrary $\operatorname{dim} \mathfrak{g} / \mathfrak{h}$ as follows: Let $V=\mathfrak{g} / \mathfrak{h}$. Let $U=\operatorname{Hom}_{K}(S(V), V)$. $U$ is injective, whence $H^{q}(V, U)=0$ for $q \geqq 1$. The calculations of Guillemin and Sternberg go through and all that remains is to identify $U$ as a graded subalgebra of $D(E)$. So let $d \in U$ and $u \in E$. If $a, b \in S(V)$, form $(d \square u)(a \otimes b)=u(b d(a)) . d \square u \in$ $\operatorname{Hom}_{K}(S(V) \otimes S(V), K)$. Set $d u=(d \square u) \circ \Delta$. One easily checks that $d: u \rightarrow d u$ belongs to $D(E) ; d$ gives a homogeneous derivation of degree $p$ if and only if $d$ is homogeneous of degree $p+1$ as a member of $U$. If $\operatorname{dim} V<\infty$, then $U \simeq E \otimes V$, which is Rim's ambient Lie algebra.

(3) If char $K=p>0, D(E)$ contains derivations of arbitrarily large negative degree, because $d^{p}$ is a derivation whenever $d$ is. For the same reason $D_{-1}(E)$ contains "nonstandard" derivations such as $u \bar{\gamma}(y)^{p}$ where $u \in E_{p-1}$ and $y \in \mathrm{g}$. These derivations do not belong to $U$. Thus our uniqueness result is stronger than Rim's.

Using their Realization Theorem, Guillemin, Spencer, and Sternberg have defined a formal notion of "induced representation" [3a]. It is motivated by the following observations: Let $G, H$, and $V$ be as in Proposition 5. Let $H^{0}=\{(\xi, \xi): \xi \in H\}$ and set $\xi^{0}=(\xi, \xi)$ for $\xi \in H$. Make $V$ into an $H^{0}$-representation space by having each $\xi^{0} \in H^{0}$ act as the identity operator. Regard $H$ and $G$ as subgroups of $H \times G$. 
Then $C_{H}^{\infty}(G, V)$ is naturally isomorphic to the space of $f \in C_{H^{0}}^{\infty}(H \times G, V)$ for which $(\zeta f)(\xi, \alpha)=L_{\zeta}^{-1} f(\xi, \alpha)$ for all $\zeta, \xi \in H$ and $\alpha \in G$ via the $\operatorname{map} f \rightarrow \bar{f}$ where $\bar{f}(\alpha)=f(\varepsilon, \alpha)$. We have $(\alpha f)^{-}=\alpha \bar{f}$ for $\alpha \in G$.

This leads to the Guillemin-Spencer-Sternberg definition: Let $\mathfrak{g}$ be a finite dimensional Lie algebra over a field $K$, let $\mathfrak{h}$ be a subalgebra of $\mathfrak{g}$, and let $V$ be a finite dimensional $\mathfrak{h}$-module. Let $\mathfrak{h}^{0}=\{(h, h): h \in \mathfrak{h}\}$ and set $h^{0}=(h, h)$ for $h \in \mathfrak{h}$. If we assume $\mathfrak{h} \cap \mathfrak{c}=\{0\}$, where $\mathfrak{c}$ is the center of $\mathfrak{g}$, then $\mathfrak{h}^{0}$ contains no nontrivial ideals of $\mathfrak{h} \oplus \mathfrak{g}$. We may therefore realize $\mathfrak{h} \oplus \mathfrak{g}$ as a Lie algebra of derivations of $S\left(\mathfrak{h} \oplus \mathfrak{g} / \mathfrak{h}^{0}\right)^{*}$. Regard $\mathfrak{h}$ and $\mathfrak{g}$ as subalgebras of $\mathfrak{h} \oplus \mathfrak{g}$. Let $W$ consist of those $w \in S\left(\mathfrak{h} \oplus \mathfrak{g} / \mathfrak{h}^{0}\right)^{*} \otimes V$ which are annihilated by $h \otimes I+I \otimes h, h \in \mathfrak{h} . W$ is invariant under $g$ and is called the $g$-module "induced" by $V$.

Observe that to make this construction we need:

(1) $\operatorname{dim} V<\infty$,

(2) $\mathfrak{h} \cap \mathfrak{c}=\{0\}$, and

(3) $\operatorname{dim} \mathfrak{g}=\operatorname{dim}\left(\mathfrak{g} \oplus \mathfrak{h} / \mathfrak{h}^{0}\right)<\infty$.

Using Remark (2) above, restriction (3) is inessential to their method. Our construction of produced module needs none of these restrictions, yet is equivalent to the "induced" construction when these restrictions hold. This follows from Theorem 1 and

Proposition 9. Let $\mathfrak{g}, \mathfrak{h}, V$, and $K$ be arbitrary. Let $\mathfrak{h}^{0}$ act trivially on $V$. Let $W$ be the subspace of $w \in \operatorname{Hom}_{\mathfrak{U}\left(\mathfrak{G}^{0}\right)}(\mathfrak{U}(\mathfrak{h} \oplus \mathfrak{g}), V)$ such that $(h w)(c)=-h w(c)$ for all $h \in \mathfrak{h}$ and $c \in \mathfrak{U}(\mathfrak{h} \oplus \mathfrak{g})$. W is $\mathfrak{g}$-invariant. If $w \in W$, let $\bar{w}$ be the restriction of $w$ to $\mathfrak{u}(\mathfrak{g})$ (regarded as a subalgebra of $\mathfrak{u}(\mathfrak{h} \oplus \mathfrak{g})$ ). Then - is a $\mathfrak{u}(\mathfrak{g})$-isomorphism of $W$ onto $\operatorname{Hom}_{\mathfrak{U}_{(\mathfrak{h})}}(\mathfrak{U}(\mathfrak{g}), V)$.

Proof. We identify $\mathfrak{u}(\mathfrak{h} \oplus \mathfrak{g})$ with $\mathfrak{u}(\mathfrak{h}) \otimes \mathfrak{u}(\mathfrak{g})$. Note that $\mathfrak{u}\left(\mathfrak{h}^{0}\right)=\Delta \mathfrak{u}(\mathfrak{h})$. Let $w \in \operatorname{Hom}_{K}(\mathfrak{H}(\mathfrak{h} \oplus \mathfrak{g}), V)$. Then $\quad w \in \operatorname{Hom}_{\mathfrak{U}_{\left(\mathfrak{h}^{0}\right)}}(\mathfrak{H}(\mathfrak{h} \oplus \mathfrak{g}), V)$ if and only if $w(\Delta(h) c)=0$ for all $h \in \mathfrak{h}$ and $c \in \mathfrak{U}(\mathfrak{h}) \otimes \mathfrak{U}(\mathfrak{g})$; i.e. if and only if $w(h z \otimes a)=$ $w\left(z \otimes h^{\prime} a\right)$ for all $h \in \mathfrak{h}, z \in \mathfrak{U}(\mathfrak{h})$, and $a \in \mathfrak{u}(\mathfrak{g})$. This condition is the same as requiring $w(z \otimes a)=w\left(1 \otimes z^{\prime} a\right)$. Therefore $w$ is determined by its values on $\mathfrak{u}(\mathfrak{g})$ and ${ }^{-}$is injective.

Let $w \in \operatorname{Hom}_{\left.\mathfrak{U}_{(\mathfrak{G}}\right)^{0}}(\mathfrak{U}(\mathfrak{h} \oplus \mathfrak{g}), V)$. Then $w \in W$ if and only if $(z w)(c)=z^{\prime} w(c)$ for all $z \in \mathfrak{U}(\mathfrak{h})$ and $c \in \mathfrak{U}(\mathfrak{h}) \otimes \mathfrak{U}(\mathfrak{g})$. But $\bar{w}(z a)=w(1 \otimes z a)=w\left(z^{\prime} \otimes a\right)=\left(z^{\prime} w\right)(1 \otimes a)$ while $z \bar{w}(a)=z w(1 \otimes a)$. Therefore $w \in W$ if and only if $\bar{w} \in \operatorname{Hom}_{\mathfrak{U}_{(\mathfrak{g})}}(\mathfrak{U}(\mathfrak{g}), V)$. Again, $(b \bar{w})(a)=\bar{w}(a b)=w(1 \otimes a b)=w((1 \otimes a)(1 \otimes b))=(b w)(1 \otimes a)=(b w)^{-}(a)$ for $a, b \in \mathfrak{U}(\mathfrak{g})$. Therefore ${ }^{-}$is a $\mathfrak{u}(\mathfrak{g})$-homomorphism. Finally, let $u \in \operatorname{Hom}_{\mathfrak{U}(\mathfrak{g})}(\mathfrak{U}(\mathfrak{g}), V)$. Set $w(z \otimes a)=u\left(z^{\prime} a\right)$. Then $w \in W$ and $\bar{w}=u$, so that - is surjective.

5. Systems of imprimitivity. Let $\mathfrak{g}$ be a Lie algebra over a field $K$ of arbitrary characteristic and let $W$ be a $g$-module. Let $\mathfrak{h}$ be a subalgebra of $\mathfrak{g}$. Let

$$
F=\operatorname{Hom}_{\mathfrak{U}(\mathfrak{G})}(\mathfrak{H}(\mathfrak{g}), K) .
$$


Definition. A (transitive) system of imprimitivity based on $\mathfrak{g} / \mathfrak{h}$ for $W$ is an $F$ module structure on $W$ such that $x(f w)=(x f) w+f(x w)$ for all $x \in \mathfrak{g}, f \in F$, and $w \in W$.

This definition is an infinitesimalization, due to Guillemin and Sternberg, of the notion of system of imprimitivity for groups introduced by Mackey [7] (see [1] for a group definition closer in spirit to the Lie algebra definition).

Proposition 10. Let $V$ be an $\mathfrak{h}$-module and let $W$ be the g-module

$$
\operatorname{Hom}_{\mathfrak{U}(\mathfrak{h})}(\mathfrak{U}(\mathfrak{g}), V) \text {. }
$$

Let $F$ act on $W$ by means of $\otimes$. The resulting F-module structure on $W$ is a system of imprimitivity based on $\mathfrak{g} / \mathfrak{h}$.

Proof. Immediate from Proposition 3.

In what follows, we shall denote the product $\otimes$ in $F$ and between $F$ and other produced representations by simple juxtaposition. The product $X$ will continue to be denoted by $x$.

Our goal is to prove a sort of converse to Proposition 8 . Let $W$ be a g-module equipped with a system of imprimitivity based on $\mathfrak{g} / \mathfrak{h}$. Set $W_{p}=F_{p} W$ for each integer $p$. Then $\left\{W_{p}\right\}$ is a filtration on $W$ with $W_{p}=W$ for $p \leqq 0$. Moreover, $F_{p} W_{q} \subseteq W_{p+q}$. Again, $\mathfrak{g} F_{p} \subseteq F_{p-1}$ and $\mathfrak{h} F_{p} \subseteq F_{p}$ according to Theorem 1. Therefore $\mathfrak{g} W_{p} \subseteq W_{p-1}$ and $\mathfrak{h} W_{p} \subseteq W_{p}$.

Lemma 3. If $w \in W_{p}$ for some $p \geqq 1$ and if $\mathrm{g} w \in W_{p}$, then $w \in W_{p+1}$.

Proof. Write

$$
\left.w=\sum_{j}^{k} f_{j} w_{j} \quad \text { (modulo } W_{p+1}\right)
$$

where $f_{j} \in F_{p}$ and $\left\{w_{j}\right\}$ is linearly independent in $W$ (modulo $W_{1}$ ). We have

$$
y_{1} w=\sum_{j}^{k}\left(y_{1} f_{j}\right) w_{j} \quad\left(\text { modulo } W_{p}\right)
$$

where $y_{1} \in \mathfrak{g}$. Iterating,

$$
y_{q} \cdots y_{1} w=\sum_{j}^{k}\left(y_{q} \cdots y_{1} f_{j}\right) w_{j} \quad\left(\operatorname{modulo} W_{p-q+1}\right) .
$$

Hence if $a \in \mathfrak{U}_{p}(\mathfrak{g})$,

$$
a w=\sum_{j}^{k}\left(a f_{j}\right) w_{j} \quad\left(\text { modulo } W_{1}\right)
$$

But

$$
\sum_{j}^{k}\left(a f_{j}\right) w_{j}=\sum_{j}^{k} f_{j}(a) w_{j} \quad\left(\text { modulo } W_{1}\right)
$$

and $a w \in W_{p} \subseteq W_{1}$. Therefore

$$
\sum_{j}^{k} f_{j}(a) w_{j}=0 \quad\left(\text { modulo } W_{1}\right)
$$

whence $f_{j}(a)=0, j=1, \ldots, k$ for all $a \in \mathfrak{U}_{p}(\mathfrak{g})$. This says that $f_{j} \in F_{p+1}, j=1, \ldots, k$ and so $w \in W_{p+1}$. 
Assume that the filtration on $W$ is separated. It follows from Lemma 3 that $V=W_{0} / W_{1} \neq\{0\} . V$ is an $\mathfrak{h}$-module and the projection $\sigma$ of $W$ on $V$ is an h-homomorphism. Let $U=\operatorname{Hom}_{\mathfrak{U}(\mathfrak{G})}(\mathfrak{u}(\mathfrak{g}), V)$ and let $\theta$ be the usual $\mathfrak{g}$-homomorphism of $W$ into $U$ defined by $(\theta w)(a)=\sigma(a w), a \in \mathfrak{u}(\mathfrak{g})$.

LEMMA 4. $w \in W_{p}$ if and only if $\theta w \in U_{p}$.

Proof. Let $w \in W_{p}$. If $a \in \mathfrak{U}_{p-1}(\mathfrak{g}), a w \in W_{1}$ so that $\sigma(a w)=0$. Therefore $\theta w \in U_{p}$. We show by induction that for all $p, \theta w \in U_{p}$ implies $w \in W_{p}$. This is true for $p=1$. Suppose it is true for $p$ and let $\theta w \in U_{p+1}$. Then $\theta w \in U_{p}$ so that $w \in W_{p}$. Again $\theta(x w)=x \theta w \in U_{p}$ for all $x \in \mathfrak{g}$. Thus $\mathfrak{g} w \in W_{p}$. By Lemma $3, w \in W_{p+1}$.

COROllary. $\theta$ is injective.

LEMMA 5. $\theta$ is an F-homomorphism.

Proof. The argument is essentially that of the last paragraph of the proof of Theorem 1. Form the $\mathfrak{u}(\mathfrak{g}) \otimes \mathfrak{u}(\mathfrak{g})$-modules $F \times W$ and $F \times U$. Define $\nu: F \times W \rightarrow W$ by $\nu(f \times x)=f w$. The formula $x(f w)=(x f) w+f(x w)$ implies the formula $a v(g)$ $=\nu(\Delta(a) g)$ for $a \in \mathfrak{U}(\mathfrak{g})$ and $g \in F \times W$. Moreover, the definition of $W_{1}$ implies $\sigma(f w)=\sigma(f(1) w)$ for $f \in F$ and $w \in W$. If $a, b \in \mathfrak{U}(\mathfrak{g}), f \in F$, and $w \in W$, then

$$
\begin{aligned}
(f \times \theta(w))(a \otimes b) & =f(a) \sigma(b w)=\sigma(f(a) b w) \\
& =\sigma((a f)(1) b w)=\sigma((a f)(b w)) \\
& =\sigma(\nu((a \otimes b)(f \times w))) .
\end{aligned}
$$

Hence $(f \times \theta(w))(c)=\sigma(\nu(c(f \times w)))$ for $c \in \mathfrak{U}(\mathfrak{g}) \otimes \mathfrak{U}(\mathfrak{g})$. Therefore

$$
\begin{aligned}
(f \theta(w))(a) & =(f \times \theta(w))(\Delta(a))=\sigma(\nu(\Delta(a)(f \times w))) \\
& =\sigma(a v(f \times w))=\sigma(a(f w))=\theta(f w)(a)
\end{aligned}
$$

for all $a \in \mathfrak{U}(\mathfrak{g})$.

Let $\phi$ be the h-homomorphism of $U$ onto $V$ defined by $\phi u=u(1)$.

Lemma 6. Let $X$ be an F-stable subspace of $U$ such that $\phi(X)=V$. Suppose either (1) $X$ is closed in the finite-open topology of $U$ ( $V$ is discrete!) or (2) $\operatorname{dim} V<\infty$. Then $X=U$.

Proof. Let $I$ be an ordered index set for $\mathfrak{g} / \mathfrak{h}$, choose $x \in \mathfrak{g}^{I}$, define $Z \subseteq J^{I}$, and define $x^{m}$ for $m \in Z$ as in $\S 2$. We shall write $l \geqq m$ for $l, m \in Z$ whenever $l_{i} \geqq m_{i}$ for all $i \in I$. Define $f_{m} \in F$ for $m \in Z$ by the formula $f_{m}\left(x^{l}\right)=\delta_{l m}$. If $u \in U$, then Lemma 2 shows that $\left(f_{m} u\right)\left(x^{l}\right)=0$ unless $l \geqq m$ and that $\left(f_{m} u\right)\left(x^{m}\right)=u(1)$. If $\left\{u_{m}\right\}_{m \in Z}$ is in $U$, then $\sum_{m} f_{m} u_{m}$ converges in the finite-open topology of $U$ in the unordered sense since it converges on every $x^{l}, l \in Z$.

Choose a linear map $\alpha: V \rightarrow X$ such that $\phi \circ \alpha$ is the identity map. Let $u$ be given in $U$. We define inductively a family $\left\{w_{m}\right\}_{m \in Z}$ in $\alpha V$ in such a way that

$$
u_{p}=u-\sum_{|m| \leqq p} f_{m} w_{m} \in U_{p+1}
$$


Set $w_{0}=\alpha(u(1))$. Then $u_{0}(1)=0$ so that $u_{0} \in U_{1}$. Suppose that $\left\{w_{m}\right\}$ have been defined for $|m| \leqq p$. For $|m|=p+1$, set $w_{m}=\alpha\left(u_{p}\left(x^{m}\right)\right)$. Then $w_{m}(1)=u_{p}\left(x^{m}\right),\left(f_{m} w_{m}\right)\left(x^{k}\right)$ $=\delta_{k m} u_{p}\left(x^{m}\right)$ for $|k| \leqq p+1$,

$$
u_{p+1}\left(x^{k}\right)=u_{p}\left(x^{k}\right)-\sum_{|m|=p+1}\left(f_{m} w_{m}\right)\left(x^{k}\right)=0
$$

for $|k| \leqq p+1$, and so $u_{p+1} \in U_{p+2}$ as desired.

It follows that $u=\sum_{m \in Z} f_{m} w_{m}$. If $X$ is closed, then $u \in X$. If $\operatorname{dim} V<\infty$, choose a basis $\left\{w_{(1)}, \ldots, w_{(q)}\right\}$ for $\alpha V$. Write

$$
w_{m}=\sum_{j}^{q} \lambda_{j m} w_{(j)}
$$

with $\lambda$ 's in $K$. Define $\left\{f_{(1)}, \ldots, f_{(q)}\right\}$ in $F$ by $f_{(j)}\left(x^{m}\right)=\lambda_{j m}$. It follows easily that

$$
u=\sum_{j}^{a} f_{(j)} w_{(j)}
$$

and once again $u \in X$. In each case, therefore, $X=U$.

We finally arrive at our main result on imprimitivity.

THEOREM 2. $\theta$ is an injective $\mathfrak{g}$ - and F-homomorphism of $W$ into $U$. If (1) $\operatorname{dim} \mathfrak{g} / \mathfrak{h}$ $<\infty$ and $W$ is complete or if (2) $\operatorname{dim} V<\infty, \theta$ is a bijection.

Proof. It remains to consider the surjectiveness of $\theta$. Let $X=\theta W . X$ is $F$-stable and $\phi X=V$. Case (2) follows from case (2) of Lemma 6, case (1) from case (1) of the lemma together with the observations that $\theta$ is filtration preserving (Lemma 4) and that the filtration and finite-open topologies on $U$ agree when $\operatorname{dim} \mathfrak{g} / \mathfrak{h}<\infty$.

ReMARK. (1) This theorem is an analogue of Mackey's Theorem 2 in [7].

(2) Guillemin, Spencer, and Sternberg [3a] have proved an imprimitivity theorem for situations in which their Realization Theorem can be used to define their "induced" representations. Our results generalize theirs.

6. An irreducibility theorem. In Theorem 8.1 of [8] Mackey has proved a criterion for the irreducibility of induced representations of group extensions. We will prove an analogue of part of this result for induced and produced representations of Lie algebra extensions.

Let $\mathfrak{g}$ be a Lie algebra and let $\mathfrak{f}$ be an ideal of $\mathfrak{g}$. Let $V$ be a $\mathfrak{f}$-module. Let $\mathfrak{h}=\left\{y \in \mathfrak{g}: \exists s \in \operatorname{Hom}_{K}(V, V) \ni:[y, k] v=s k v-k s v\right.$ for all $v \in V$ and $\left.k \in \mathfrak{t}\right\}$. Clearly $\mathfrak{h}$ is a subalgebra of $\mathfrak{g}$ and $\mathfrak{t} \subseteq \mathfrak{h}$.

Definition. A module is called absolutely irreducible if it is irreducible under arbitrary extensions of the base field $K$, or equivalently if the commuting ring consists of multiplications by $K$.

THEOREM 3. (a) If $\mathfrak{h} \neq \mathfrak{f}$, then $\mathfrak{u}(\mathfrak{g}) \otimes \mathfrak{u}(\mathfrak{t}) V$ is reducible.

(b) Suppose $K$ has characteristic 0 and $V$ is absolutely irreducible. Let $W$ be an

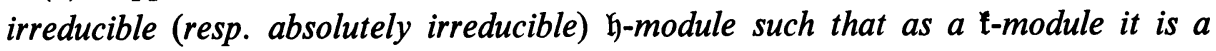


direct sum of copies of $V$. Then $\mathfrak{u}(\mathfrak{g}) \otimes \mathfrak{u}_{(\mathfrak{h})} W$ is irreducible (resp. absolutely irreducible).

For the proof of this theorem, we need the following lemma:

Lemma 7. For $x \in \mathfrak{g}$, let $\delta(x) k=[k, x]$ for $k \in \mathfrak{f}$. $\delta$ is an antihomomorphism of $\mathfrak{g}$ into the Lie algebra of derivations of $\mathfrak{t}$. Extend $\delta(x)$ to be a derivation of $\mathfrak{H}(\mathfrak{t})$. Then extend $\delta$ to be an antihomomorphism of $\mathfrak{u}(\mathfrak{g})$ into $\operatorname{Hom}_{K}(\mathfrak{u}(\mathfrak{t}), \mathfrak{u}(\mathfrak{t}))$. Let $z \in \mathfrak{H}(\mathfrak{t})$ and $y_{1}, \ldots, y_{p} \in \mathfrak{g}$. Then

$$
z y_{1}^{r_{1}} \cdots y_{p}^{r_{p}}=\sum_{0 \leqq k_{i} \leqq r_{i}}\left(\begin{array}{c}
r_{1} \\
k_{1}
\end{array}\right) \cdots\left(\begin{array}{c}
r_{p} \\
k_{p}
\end{array}\right) y_{1}^{k_{1}} \cdots y_{p}^{k_{p}}\left[\delta\left(y_{1}^{r_{1}-k_{1}} \cdots y_{p}^{r_{p}-k_{p}}\right) z\right] .
$$

Proof. We shall prove this in the case $r_{1}=\cdots=r_{p}=1$. The general result will follow by identifying $y$ 's and collecting terms exactly as in Lemma 2 . The lemma holds trivially for $p=0$. Suppose it holds for $p$. Then

$$
\begin{aligned}
z y_{1} \cdots y_{p+1}= & y_{1} z y_{2} \cdots y_{p+1}+\left[\delta\left(y_{1}\right) z\right] y_{2} \cdots y_{p+1} \\
= & y_{1} \sum_{0 \leqq k_{i} \leqq 1} y_{2}^{k_{2}} \cdots y_{p+1}^{k_{p+1}}\left[\delta\left(y_{2}^{1-k_{2}} \cdots y_{p+1}^{1-k_{p+1}}\right) z\right] \\
& +\sum_{0 \leqq k_{i} \leqq 1} y_{2}^{k_{2}} \cdots y_{p+1}^{k_{p+1}}\left[\delta\left(y_{2}^{1-k_{2}} \cdots y_{p+1}^{1-k_{p+1}}\right) \delta\left(y_{1}\right) z\right] \\
= & \sum_{0 \leqq k_{i} \leqq 1} y_{1}^{k_{1}} y_{2}^{k_{2}} \cdots y_{p+1}^{k_{p+1}}\left[\delta\left(y_{1}^{1-k_{1}} y_{2}^{1-k_{2}} \cdots y_{p+1}^{1-k_{p+1}}\right) z\right]
\end{aligned}
$$

because $\delta(a) \delta(b)=\delta(b a)$.

Proof of theorem. (a) Choose $0 \neq y \in \mathfrak{h}, y \notin \mathfrak{t}$. Choose an ordered index $I$ with highest element $i_{0}$ and $x \in \mathfrak{g}^{I}$ so that $\left\{x_{i}\right\}_{i \in I}$ is a basis for $\mathfrak{g}$ (modulo $\mathfrak{t}$ ) and so that $x_{i_{0}}=y$. Define $Z \subseteq J^{I}$ and $x^{m}$ for $m \in Z$ as in $\S 2$. Set $U=\mathfrak{U}(\mathfrak{g}) \otimes \mathfrak{u}_{(\mathfrak{k})} V$ and filter it as in $\$ 2$. Choose $s \in \operatorname{Hom}_{K}(V, V)$ for $y$ as in the definition of $\mathfrak{h}$. Define $r \in Z$ by $r_{i}=\delta_{i i_{0}}$. We add and subtract in $Z$ in the usual way. Let $T$ be the subspace of $U$ consisting of all linear combinations of elements of the form $x^{m+r} \otimes v-x^{m} \otimes s v$ for $m \in Z$ and $v \in V$. Since the map ` (see $\S 2)$ of $S(\mathrm{~g} / \mathrm{t}) \otimes V$ into $U$ is a linear isomorphism, we see that $T \cap U_{0}=\{0\}$ and hence that $T$ is a nonvoid proper subspace of $U$. We finish by showing that $T$ is $\mathfrak{u}(\mathfrak{g})$-invariant. Let $k \in \mathfrak{f}$. Then

$$
\begin{aligned}
k\left(x_{i_{0}} \otimes v-1 \otimes s v\right) & =\left[k, x_{i_{0}}\right] \otimes v+x_{i_{0}} k \otimes v-k \otimes s v \\
& =x_{i_{0}} \otimes k v-1 \otimes\left(k s v+\left[x_{i_{0}}, k\right] v\right) \\
& =x_{i_{0}} \otimes k v-1 \otimes s k v
\end{aligned}
$$

by the definition of $s$. Thus $S=\left\{x_{\mathfrak{i}_{0}} \otimes v-1 \otimes s v: v \in V\right\}$ is $\mathfrak{t}$ - and hence $\mathfrak{u}(\mathfrak{t})$ invariant. But $\mathfrak{U}(\mathfrak{g})=\sum_{m \in Z} x^{m} \mathfrak{u}(\mathfrak{t})$. Thus $\mathfrak{U}(\mathfrak{g}) S=\sum_{m \in Z} x^{m} S=T$ because $x^{m} x_{\mathfrak{i}_{0}}=x^{m+r}$. Therefore $T$ is $\mathfrak{u}(\mathfrak{g})$-invariant and $U$ is reducible.

(b) Let $\left\{x_{\mathfrak{i}}\right\}_{i \in I}$ be an ordered basis for $\mathfrak{g}$ (modulo $\mathfrak{h}$ ) as in $\S 2$. Set $U=\mathfrak{u}(\mathfrak{g}) \otimes \mathfrak{u}_{(\mathfrak{g})} W$ and filter it as in $\S 2$. Let $T$ be a non- $\{0\} \mathfrak{u}(\mathfrak{g})$-invariant subspace of $U$. We will show that $T=U$. 
Case I. Suppose that $T \cap U_{0} \neq\{0\}$. Then $\mathfrak{u}(\mathfrak{h})\left(T \cap U_{0}\right)=1 \otimes W$ by the irreducibility of $W$ and the fact that $h(1 \otimes w)=1 \otimes h w$ for $h \in \mathfrak{h}$ and $w \in W$. Therefore $T \supseteq \sum_{m \in Z} x^{m} \otimes W=U$.

Case II. Suppose that $T \cap U_{0}=\{0\}$. Let $p$ be the least integer such that $T \cap U_{p} \neq\{0\} . p \geqq 1$. Choose $0 \neq u \in T \cap U_{p}$. Remembering that $\iota$ is a filtration preserving isomorphism, we write

$$
u=\sum_{|m| \leqq p} x^{m} \otimes w_{m}
$$

with $w_{m} \in W$. By hypothesis, $W=\dot{+}_{\alpha} V_{\alpha}$, where the $V_{\alpha}$ are $\mathrm{t}$-submodules of $W$ $\mathrm{t}$-isomorphic to $V$. Only a finite number of the $w_{m}$ are nonzero and hence the $w_{m}$ have components in only a finite number of the $V_{\alpha}$, say $V_{\alpha_{1}}, \ldots, V_{\alpha_{q}}$. Let $\zeta^{n}$, $n=1, \ldots, q$, be a $\mathfrak{t}$-homomorphism of $W$ into $V$ which vanishes on $\sum_{\alpha \neq \alpha_{n}} V_{\alpha}$ and is an isomorphism on $V_{\alpha_{n}}$. Then, if $z \in \mathfrak{u}(\mathfrak{t}), z w_{m}=0$ if and only if $z \zeta^{n}\left(w_{m}\right)=0$ for all $n=1, \ldots, q$.

Choose $0 \neq v \in V$. Without loss of generality we claim we may assume that $\zeta^{n} w_{m}=\lambda_{m}^{n} v$ for $|m|=p$ and all $n$, the $\lambda$ 's $\in K$ and not all 0 . In fact, for our original $u$ and the corresponding $w$ 's, we may use the Chevalley-Jacobson density theorem on the absolutely irreducible $\mathfrak{t}$-module $V$ to find $z_{0} \in \mathfrak{U}(\mathfrak{f})$ such that $z_{0} \zeta^{n} w_{m}=\lambda_{m}^{n} v$ where the $\lambda$ 's are as above. It follows from Lemma 7 that

$$
z_{0} u=\sum_{|m|=p} x^{m} \otimes z_{0} w_{m}
$$

(modulo $U_{p-1}$ ). Using $z_{0} u$ instead of our original $u$, we have an element of $T \cap U_{p}$ satisfying our assumptions.

For all $z \in \mathfrak{U}(\mathfrak{t})$ and $|m|=p, \zeta^{n}\left(z w_{m}\right)=\lambda_{m}^{n} z v$. Therefore $w_{m}=0,|m|=p$ if and only if $\lambda_{m}^{n}=0$ for all $n$. Moreover, if $w_{m} \neq 0$, then $z w_{m}=0$ if and only if $z v=0$.

Choose $m$ so that $|m|=p$ and $w_{m} \neq 0$. For each $i \in I$ define $r(i) \in Z$ by $r(i)_{j}=\delta_{i j}$. Choose $i_{0} \in I$ with $m_{i_{0}}>0$. Set $l=m-r\left(i_{0}\right)$. Using Lemma 6 again, we see that the coefficient of $x^{l}$ in $\iota^{-1}(z u)$ is

$$
z w_{l}-\sum_{j \in I}\left(l_{j}+1\right)\left[\delta\left(x_{j}\right) z\right] w_{l+r(j)} .
$$

Choose $n_{0}$ so that $\lambda_{m}^{n_{0}} \neq 0$. Applying $\zeta^{n_{0}}$ to (1) we get $z v_{0}-[\delta(y) z] v$, where $v_{0}=\zeta^{n_{0}} w_{l}$ and

$$
y=\sum_{j \in I}\left(l_{j}+1\right) \lambda_{l+r(j)}^{n_{0}} x_{j} .
$$

The coefficient of $x_{i_{0}}$ in $y$ is $\lambda_{m}^{n_{0}}$ and hence $y \notin \mathfrak{h}$.

We now prove that $y \in \mathfrak{h}$, which contradiction will show that Case II is void. Every member of $V$ is of the form $z v$. Suppose $z v=0$. Since

$$
z u=\sum_{|k|=m} x^{k} \otimes z w_{k} \quad \text { (modulo } U_{p-1} \text { ) }
$$

and $\zeta^{n} z w_{k}=\lambda_{k}^{n} z v$ for $1 \leqq n \leqq q$, we see that $z u \in T \cap U_{p-1}$. By the minimal choice of $p, z u=0$. Hence (1) vanishes and so $z v_{0}-[\delta(y) z] v=0$. Therefore we may define an 
operator $s \in \operatorname{Hom}_{K}(V, V)$ by $s z v=z v_{0}-[\delta(y) z] v$. Suppose $k \in \mathfrak{t}$. Then

$$
\begin{aligned}
s k(z v)-k s(z v) & =k z v_{0}-[\delta(y)(k z)] v-\left\{k z v_{0}-k[\delta(y) z] v\right\} \\
& =k z v_{0}-[(\delta(y) k) z+k \delta(y) z] v-k z v_{0}+[k \delta(y) z] v \\
& =[\delta(y) k] z v=[k, y] z_{v} .
\end{aligned}
$$

Therefore $y \in \mathfrak{h}$.

To get absolute irreducibility in (b) it suffices to consider arbitrary field extensions of $K$ and apply what we have already proved.

Using Proposition 1 and the Corollary and remarks following it, we can dualize Theorem 3 and obtain

THEOREM 4. Let $V$ be a finite dimensional $\mathfrak{t}$-module over the topological field $K$. Give $V$ the weak topology of the pairing $\left(V, V^{*}\right)$. Define $\mathfrak{h}$ as in Theorem 3.

(a) If $\mathfrak{h} \neq \mathfrak{f}$, then $\operatorname{Hom}_{\mathfrak{U}(\mathfrak{g})}(\mathfrak{U}(\mathfrak{g}), V)$ is topologically reducible in the finite-open topology.

(b) Suppose $K$ has characteristic 0 and $V$ is absolutely irreducible. Let $W$ be a topological vector space over $K$ which is an $\mathfrak{h}$-module such that the elements of $\mathfrak{h}$ act continuously. Suppose that as $a \mathrm{t}$-module, $W$ is topologically and $\mathrm{t}$-module isomorphic to a complete direct product of copies of $V$. Then $\operatorname{Hom}_{\mathfrak{u}(\mathfrak{G})}(\mathfrak{u}(\mathfrak{g}), W)$ is topologically irreducible in the finite-open topology.

Proof. (a) $\operatorname{Hom}_{\mathfrak{U}(\mathfrak{h})}(\mathfrak{u}(\mathfrak{g}), V) \simeq\left(\mathfrak{u}(\mathfrak{g}) \otimes \mathfrak{u}_{(\mathfrak{h})} V^{*}\right)^{*}$. The subalgebra $\mathfrak{h}$ defined by the $\mathfrak{f}$-module $V^{*}$ is the same as the one defined by $V$. Moreover $V^{* *} \simeq V$. Apply Theorem 3, Proposition 1, and its Corollary.

(b) Suppose that as a $\mathfrak{f}$-module $W=\Pi V_{\alpha}$. Let $L=\sum V_{\alpha}^{*}$. Then as a $\mathfrak{t}$-module $W=L^{*}$ with the usual canonical identifications. Moreover the topology of $W$ is the weak topology of the pairing $(L, W)$. Now each $h \in \mathfrak{h}$ acts continuously on $W$. Therefore it is the transpose of a unique linear map $\omega(h)$ on $L$. For $h \in \mathfrak{h}$ and $l \in L$, set $h l=-\omega(h) l$. One readily checks that this makes $L$ an $\mathfrak{h}$-module and that $W=L^{*}$ as $\mathfrak{h}$-modules. The $\mathfrak{t}$-module structure that $\omega$ defines on $L$ is the original $\mathfrak{t}$-module structure on $L$. It is now easy to check that the hypothesis of Theorem 3(b) are satisfied for $V^{*}$ and $L$. Therefore $\mathfrak{u}(\mathfrak{g}) \otimes \mathfrak{u}_{(\mathfrak{g})} L$ is irreducible. Since

$$
\operatorname{Hom}_{\mathfrak{u}(\mathfrak{h})}(\mathfrak{u}(\mathfrak{h}), W) \simeq\left(\mathfrak{u}(\mathfrak{g}) \otimes \mathfrak{u}_{(\mathfrak{h})} L\right)^{*},
$$

our result follows.

REMARK. One would like to have a Lie algebra analogue for the second part of Mackey's theorem. However, there seem to be fundamental obstacles in the way of carrying this out. For example, in [2] Dixmier has constructed certain irreducible representations of nilpotent Lie algebras which are not induced from one-dimensional representations of subalgebras.

\section{REFERENCES}

1. Robert J. Blattner, Positive definite measures, Proc. Amer. Math. Soc. 14 (1963), 423-428.

2. J. Dixmier, Représentations irréductibles des algèbres de Lie nilpotentes, An. Acad. Brasil. Ci. 35 (1963), 491-519. 
2a. J. Dixmier, Représentations irréductibles des algèbres de Lie résolubles, J. Math. Pures Appl. 45 (1966), 1-66.

3. Victor W. Guillemin and Shlomo Sternberg, An algebraic model of transitive differential geometry, Bull. Amer. Math. Soc. 70 (1964), 16-47.

3a. Victor W. Guillemin, D. C. Spencer and Shlomo Sternberg, Representation theory of transitive Lie algebras. I: The Mackey imprimitivity theorem and its generalization, (unpublished).

4. Harish-Chandra, On some applications of the universal enveloping algebra of a semisimple Lie algebra, Trans. Amer. Math. Soc. 70 (1951), 28-96.

5. —_, Representations of a semi-simple Lie group on a Banach space. I, Trans. Amer. Math. Soc. 75 (1953), 185-243.

6. D. G. Higman, Induced and produced modules, Canad. J. Math. 7 (1955), 490-508.

7. G. W. Mackey, Imprimitivity for representations of locally compact groups. I, Proc. Nat. Acad. Sci. U.S.A. 35 (1949), 537-545.

8. - Unitary representations of group extensions. I, Acta Math. 99 (1958), 265-311.

9. D. S. Rim, Deformations of transitive Lie algebras, Ann. of Math. (2) 83 (1966), 339-357.

10. Séminaire "Sophus Lie" 1955-1956, Secrétariat mathématique, Paris, 1957.

11. I. M. Singer and Shlomo Sternberg, The infinite groups of Lie and Cartan. I: The transitive groups, J. Analyse Math. 15 (1965), 1-114.

12. Nolan R. Wallach, Induced representations of Lie algebras and a theorem of Borel-Weil, Trans. Amer. Math. Soc. 136 (1969), 181-187.

\author{
University of California, \\ Los Angeles, California
}

